PROCEEDINGS OF THE

AMERICAN MATHEMATICAL SOCIETY

Volume 128, Number 8, Pages 2291-2296

S 0002-9939(00)05741-5

Article electronically published on March 29, 2000

\title{
WEYL'S THEOREM HOLDS FOR ALGEBRAICALLY HYPONORMAL OPERATORS
}

\author{
YOUNG MIN HAN AND WOO YOUNG LEE
}

(Communicated by David R. Larson)

\begin{abstract}
In this note it is shown that if $T$ is an "algebraically hyponormal" operator, i.e., $p(T)$ is hyponormal for some nonconstant complex polynomial $p$, then for every $f \in H(\sigma(T))$, Weyl's theorem holds for $f(T)$, where $H(\sigma(T))$ denotes the set of analytic functions on an open neighborhood of $\sigma(T)$.
\end{abstract}

H. Weyl [12] examined the spectra of all compact perturbations $T+K$ of a single hermitian operator $T$ and discovered that $\lambda \in \sigma(T+K)$ for every compact operator $K$ if and only if $\lambda$ is not an isolated eigenvalue of finite multiplicity in $\sigma(T)$. Today this result is known as Weyl's theorem, and it has been extended from hermitian operators to hyponormal operators and to Toeplitz operators by L. Coburn [4, and to several classes of operators including hyponormal operators by S. Berberian [1, 2]. The aim of this note is to show that Weyl's theorem holds for "algebraically hyponormal" operators.

Throughout this note let $\mathcal{L}(\mathcal{H})$ denote the algebra of bounded linear operators acting on an infinite dimensional Hilbert space $\mathcal{H}$. If $T \in \mathcal{L}(\mathcal{H})$ write $N(T)$ and $R(T)$ for the null space and range of $T ; \sigma(T)$ for the spectrum of $T ; \pi_{0}(T)$ for the set of eigenvalues of $T ; \pi_{00}(T)$ for the isolated points of $\sigma(T)$ which are eigenvalues of finite multiplicity. An operator $T \in \mathcal{L}(\mathcal{H})$ is called Fredholm if it has closed range with finite dimensional null space and its range of finite co-dimension. The index of a Fredholm operator $T \in \mathcal{L}(\mathcal{H})$ is given by

$$
\operatorname{ind}(T)=\operatorname{dim} N(T)-\operatorname{dim} R(T)^{\perp}\left(=\operatorname{dim} N(T)-\operatorname{dim} N\left(T^{*}\right)\right) .
$$

An operator $T \in \mathcal{L}(\mathcal{H})$ is called Weyl if it is Fredholm of index zero. An operator $T \in \mathcal{L}(\mathcal{H})$ is called Browder if it is Fredholm "of finite ascent and descent": equivalently ([7], Theorem 7.9.3]) if $T$ is Fredholm and $T-\lambda I$ is invertible for sufficiently small $\lambda \neq 0$ in $\mathbb{C}$. The essential spectrum $\sigma_{e}(T)$, the Weyl spectrum $\omega(T)$ and the Browder spectrum $\sigma_{b}(T)$ of $T \in \mathcal{L}(\mathcal{H})$ are defined by (cf. [6], [7])

$$
\begin{aligned}
\sigma_{e}(T) & =\{\lambda \in \mathbb{C}: T-\lambda I \text { is not Fredholm }\}, \\
\omega(T) & =\{\lambda \in \mathbb{C}: T-\lambda I \text { is not Weyl }\}, \\
\sigma_{b}(T) & =\{\lambda \in \mathbb{C}: T-\lambda I \text { is not Browder }\}
\end{aligned}
$$

Received by the editors August 22, 1998.

2000 Mathematics Subject Classification. Primary 47A10, 47A53; Secondary 47B20.

Key words and phrases. Weyl's theorem, algebraically hyponormal operators, unilateral weighted shifts.

This work was partially supported by the BSRI-97-1420 and the KOSEF through the GARC at Seoul National University. 
evidently

$$
\sigma_{e}(T) \subseteq \omega(T) \subseteq \sigma_{b}(T)=\sigma_{e}(T) \cup \operatorname{acc} \sigma(T),
$$

where we write $\operatorname{acc} \mathbf{K}$ for the accumulation points of $\mathbf{K} \subseteq \mathbb{C}$. We say that Weyl's theorem holds for $T \in \mathcal{L}(\mathcal{H})$ if there is equality

$$
\sigma(T) \backslash \omega(T)=\pi_{00}(T) .
$$

An operator $T \in \mathcal{L}(\mathcal{H})$ is called isoloid if every isolated point of $\sigma(T)$ is an eigenvalue of $T$ (cf. 2], [10]). An operator $T \in \mathcal{L}(\mathcal{H})$ will be called algebraically hyponormal if there exists a nonconstant complex polynomial $p$ such that $p(T)$ is hyponormal. Evidently the $p$ th roots of hyponormal operators (i.e., the operator $T$ such that $T^{p}$ is hyponormal for $p \in \mathbb{N}$ ) are algebraically hyponormal (see [3] for the $p$ th roots of operators). But the converse is not true in general: for example if $T=\left(\begin{array}{ll}1 & 1 \\ 0 & 1\end{array}\right)$ on $\ell_{2} \oplus \ell_{2}$, then $T^{p}$ is not hyponormal for any $p \in \mathbb{N}$, whereas $p(T)=0$ with $p(z)=(z-1)^{2}$. Recall that an operator $T \in \mathcal{L}(\mathcal{H})$ is called polynomially hyponormal if $p(T)$ is hyponormal for every complex polynomial $p$. Evidently,

polynomially hyponormal $\subseteq$ hyponormal

$$
\begin{aligned}
& \subseteq \text { the } p \text { th roots of hyponormal operators } \\
& \subseteq \text { algebraically hyponormal. }
\end{aligned}
$$

The following facts follow from the above definition and the well-known facts of hyponormal operators.

(a) If $T \in \mathcal{L}(\mathcal{H})$ is algebraically hyponormal, then so is $T-\lambda I$ for each $\lambda \in \mathbb{C}$.

(b) If $T \in \mathcal{L}(\mathcal{H})$ is algebraically hyponormal and $\mathcal{M} \subseteq \mathcal{H}$ is invariant under $T$, then $T \mid \mathcal{M}$ is algebraically hyponormal.

(c) Unitary equivalence preserves algebraic hyponormality.

The following lemma gives the essential facts for algebraically hyponormal operators that we will need to prove the main theorem.

Lemma 1. Suppose $T \in \mathcal{L}(\mathcal{H})$.

(i) If $T$ is algebraically hyponormal and quasinilpotent, then $T$ is nilpotent.

(ii) If $T$ is algebraically hyponormal, then $T$ is isoloid.

(iii) If $T$ is algebraically hyponormal, then $T$ has finite ascent.

Proof. (i) Suppose $p(T)$ is hyponormal for some nonconstant polynomial $p$. Since hyponormality is translation-invariant, we may assume $p(0)=0$. Thus we can write $p(\lambda) \equiv a_{0} \lambda^{m}\left(\lambda-\lambda_{1}\right) \cdots\left(\lambda-\lambda_{n}\right)\left(m \neq 0, \lambda_{i} \neq 0\right.$ for every $\left.1 \leq i \leq n\right)$. If $T$ is quasinilpotent, then $\sigma(p(T))=p(\sigma(T))=p(\{0\})=\{0\}$, so that $p(T)$ is also quasinilpotent. Since the only hyponormal quasinilpotent operator is zero, it follows that $a_{0} T^{m}\left(T-\lambda_{1} I\right) \cdots\left(T-\lambda_{n} I\right)=0$. Since $T-\lambda_{i} I$ is invertible for every $1 \leq i \leq n$, we have that $T^{m}=0$.

(ii) Suppose $p(T)$ is hyponormal for some nonconstant polynomial $p$. Let $\lambda \in$ iso $\sigma(T)$. Then using the spectral decomposition, we can represent $T$ as the direct sum $T=T_{1} \oplus T_{2}$, where $\sigma\left(T_{1}\right)=\{\lambda\}$ and $\sigma\left(T_{2}\right)=\sigma(T) \backslash\{\lambda\}$. By the preceding remark, $T_{1}-\lambda I$ is also algebraically hyponormal. Since $T_{1}-\lambda I$ is quasinilpotent it follows from the statement (i) that $T_{1}-\lambda I$ is nilpotent. Therefore $\lambda \in \pi_{0}\left(T_{1}\right)$ and hence $\lambda \in \pi_{0}(T)$. This shows that $T$ is isoloid.

(iii) Suppose $p(T)$ is hyponormal for some nonconstant polynomial $p$. We may assume $p(0)=0$. If $p(\lambda) \equiv a_{0} \lambda^{m}$, then $N\left(T^{m}\right)=N\left(T^{2 m}\right)$ because hyponormal 
operators are of ascent 1 . Thus we write $p(\lambda) \equiv a_{0} \lambda^{m}\left(\lambda-\lambda_{1}\right) \cdots\left(\lambda-\lambda_{n}\right)(m \neq 0$; $\lambda_{i} \neq 0$ for $\left.1 \leq i \leq n\right)$. We then claim that

$$
N\left(T^{m}\right)=N\left(T^{m+1}\right) .
$$

To show (1.1), let $x(\neq 0) \in N\left(T^{m+1}\right)$. Then we can write

$$
p(T) x=(-1)^{n} a_{0} \lambda_{1} \cdots \lambda_{n} T^{m} x .
$$

Thus we have

$$
\begin{aligned}
\left|a_{0} \lambda_{1} \cdots \lambda_{n}\right|^{2}\left\|T^{m} x\right\|^{2} & =(p(T) x, p(T) x) \\
& \leq\left\|p(T)^{*} p(T) x\right\|\|x\| \\
& \leq\left\|p(T)^{2} x\right\|\|x\| \quad \text { (because } p(T) \text { is hyponormal) } \\
& =\left\|a_{0}^{2}\left(T-\lambda_{1} I\right)^{2} \cdots\left(T-\lambda_{n} I\right)^{2} T^{2 m} x\right\|\|x\| \\
& =0
\end{aligned}
$$

which implies $x \in N\left(T^{m}\right)$. Therefore $N\left(T^{m+1}\right) \subseteq N\left(T^{m}\right)$ and the reverse inclusion is evident. This completes the proof.

Lemma 1 shows that algebraically hyponormal operators share several properties with hyponormal operators. However there exists a deep gap between those two classes of operators: for example, in a sense of spectrum, it can be compared as a gap between nilpotent and "normaloid" (i.e., norm equals spectral radius). In spite of it, we have:

Theorem 2. Weyl's theorem holds for every algebraically hyponormal operator.

Proof. Suppose $p(T)$ is hyponormal for some nonconstant polynomial $p$. We first prove that $\pi_{00}(T) \subseteq \sigma(T) \backslash \omega(T)$. Since algebraic hyponormality is translationinvariant, it suffices to show that

$$
0 \in \pi_{00}(T) \Longrightarrow T \text { is Weyl but not invertible. }
$$

Suppose $0 \in \pi_{00}(T)$. Now using the spectral projection $P=\frac{1}{2 \pi i} \int_{\partial B_{0}}(\lambda I-T)^{-1} d \lambda$, where $B_{0}$ is an open disk of center 0 which contains no other points of $\sigma(T)$, we can represent $T$ as the direct sum

$$
T=T_{1} \oplus T_{2}, \quad \text { where } \sigma\left(T_{1}\right)=\{0\} \text { and } \sigma\left(T_{2}\right)=\sigma(T) \backslash\{0\} .
$$

But then $T_{1}$ is also algebraically hyponormal and quasinilpotent. Thus by Lemma 1 (i), $T_{1}$ is nilpotent. Thus we should have that $\operatorname{dim} R(P)<\infty$ : if it were not so, then $N\left(T_{1}\right)$ would be infinite dimensional, so that $0 \notin \pi_{00}(T)$, giving a contradiction. Therefore $T_{1}$ is a finite dimensional operator. Since finite dimensional operators are always Weyl it follows that $T_{1}$ is Weyl. But since $T_{2}$ is invertible we can conclude that $T$ is Weyl. Therefore $\pi_{00}(T) \subseteq \sigma(T) \backslash \omega(T)$. For the reverse inclusion, suppose $\lambda \in \sigma(T) \backslash \omega(T)$. Thus $T-\lambda I$ is Weyl. Then by the "Index Product Theorem",

$\operatorname{dim} N\left((T-\lambda I)^{n}\right)-\operatorname{dim} R\left((T-\lambda I)^{n}\right)^{\perp}=\operatorname{ind}\left((T-\lambda I)^{n}\right)=n \operatorname{ind}(T-\lambda I)=0$.

Thus if $\operatorname{dim} N\left((T-\lambda I)^{n}\right)$ is a constant, then so is $\operatorname{dim} R\left((T-\lambda I)^{n}\right)^{\perp}$. Consequently finite ascent forces finite descent. Therefore by Lemma 1 (iii), $T-\lambda I$ is Weyl of finite ascent and descent, and thus it is Browder. Therefore $\lambda \in \pi_{00}(T)$. This completes the proof. 
It was known ([9, Theorem 1], [11, Theorem 3.6]) that the Weyl spectrum obeys the spectral mapping theorem for hyponormal operators. We can prove more:

Theorem 3. If $T \in \mathcal{L}(\mathcal{H})$ is algebraically hyponormal, then

$$
\omega(f(T))=f(\omega(T)) \quad \text { for every } f \in H(\sigma(T)),
$$

where $H(\sigma(T))$ denotes the set of analytic functions on an open neighborhood of $\sigma(T)$.

Proof. First of all we prove the equality (3.1) when $f$ is a polynomial. In view of [8, Theorem 5], it suffices to show that

$$
\text { ind }(T-\lambda I) \text { ind }(T-\mu I) \geq 0 \text { for each pair } \lambda, \mu \in \mathbb{C} \backslash \sigma_{e}(T) \text {. }
$$

By Lemma 1 (iii), $T-\lambda I$ has finite ascent for every $\lambda \in \mathbb{C}$. Observe that if $S \in \mathcal{L}(\mathcal{H})$ is Fredholm of finite ascent, then $\operatorname{ind}(S) \leq 0$ : indeed, either if $S$ has finite descent, then $S$ is Browder and hence ind $(S)=0$, or if $S$ does not have finite descent, then

$$
n \operatorname{ind}(S)=\operatorname{dim} N\left(S^{n}\right)-\operatorname{dim} R\left(S^{n}\right)^{\perp} \longrightarrow-\infty \quad \text { as } n \longrightarrow \infty,
$$

which implies that ind $(S)<0$. Thus we can see that $(3.2)$ holds for every algebraically hyponormal operator $T$. This proves that the equality (3.1) holds for every polynomial $f$. Now the equality (3.1) for $f \in H(\sigma(T))$ follows at once from an argument of Oberai [10, Theorem 2].

We now have:

Corollary 4. If $T \in \mathcal{L}(\mathcal{H})$ is algebraically hyponormal, then for every $f \in H(\sigma(T))$, Weyl's theorem holds for $f(T)$.

Proof. Remembering ([9, Lemma]) that if $T$ is isoloid, then

$$
f\left(\sigma(T) \backslash \pi_{00}(T)\right)=\sigma(f(T)) \backslash \pi_{00}(f(T)) \text { for every } f \in H(\sigma(T)) ;
$$

it follows from Lemma 1 (ii), Theorem 2 and Theorem 3 that

$$
\sigma(f(T)) \backslash \pi_{00}(f(T))=f\left(\sigma(T) \backslash \pi_{00}(T)\right)=f(\omega(T))=\omega(f(T)),
$$

which implies that Weyl's theorem holds for $f(T)$.

We next consider algebraically hyponormal weighted shifts. Let $\left\{e_{n}\right\}_{n=0}^{\infty}$ be the canonical orthonormal basis for $\ell_{2}$, let $\left\{\alpha_{n}\right\}_{n=0}^{\infty}$ be a bounded sequence of nonnegative numbers and let $W_{\alpha}$ be the (unilateral) weighted shift with the weights $\alpha=\left\{\alpha_{n}\right\}$ defined by $W_{\alpha} e_{n}:=\alpha_{n} e_{n+1}(n \geq 0)$. It is well known that $W_{\alpha}$ is hyponormal if and only if the weight sequence $\left\{\alpha_{n}\right\}$ is monotonically increasing. A straightforward calculation shows that $W_{\alpha}^{p}$ is hyponormal for $p \in \mathbb{N}$ if and only if the weight sequence $\left\{\alpha_{n}\right\}$ satisfies that for each $m=0,1, \cdots, p-1$,

$$
\prod_{j=m}^{p+m-1} \alpha_{j} \leq \prod_{j=p+m}^{2 p+m-1} \alpha_{j} \leq \prod_{j=2 p+m}^{3 p+m-1} \alpha_{j} \leq \cdots
$$

Hence, in particular, if $W_{\alpha}$ is a $p$ th root of a hyponormal operator and $\alpha=\left\{\alpha_{n}\right\}$ contains infinitely many zeros, then by (4.1), $W_{\alpha}$ can be written as an infinite direct sum of finite dimensional nilpotents of nilpotency at most $p$, which implies that $W_{\alpha}$ is nilpotent. In general the infinitely many zeros in the weights don't guarantee the nilpotence of the weighted shift $W_{\alpha}$ (see [5, Solution 98]). It however seems to be difficult to find necessary and sufficient conditions, in terms of weights, for the weighted shift $W_{\alpha}$ to be algebraically hyponormal. On the other hand, 
Weyl's theorem is not transmitted to or from adjoint operators: for example Weyl's theorem holds for the weighted shift $W_{\alpha}$ with the weights $\alpha=\left\{\frac{1}{n+1}\right\}_{n=0}^{\infty}$, but fails for its adjoint $W_{\alpha}^{*}$. We however have:

Corollary 5. If $W_{\alpha}$ is an algebraically hyponormal weighted shift, then Weyl's theorem holds for $W_{\alpha}$ and $W_{\alpha}^{*}$ both.

Proof. In view of Theorem 2, it suffices to show Weyl's theorem for $W_{\alpha}^{*}$. It is well known that the spectrum of the weighted shift is a (possibly degenerated) disc with center 0 . If $\sigma\left(W_{\alpha}\right)=\{0\}$, then by Lemma $1(\mathrm{i}), W_{\alpha}$ is nilpotent and therefore Weyl's theorem holds for $W_{\alpha}^{*}$. If instead $\sigma\left(W_{\alpha}\right) \neq\{0\}$, then iso $\sigma\left(W_{\alpha}\right)=\emptyset$, so that the result follows at once from [1, Example 4].

We conclude with a structure theorem for algebraically hyponormal compact operators.

Corollary 6. If $T \in \mathcal{L}(\mathcal{H})$ is algebraically hyponormal and compact, then $T$ is decomposed into the direct sum

$$
T=A \oplus\left(\bigoplus_{n=1}^{\infty} F_{n}\right),
$$

where

(i) $A$ is an (possibly infinite dimensional) algebraic operator;

(ii) $F_{n}$ is a finite dimensional operator for every $n=1,2, \cdots$;

(iii) $p\left(F_{n}\right)=\lambda_{n} \rightarrow 0$ as $n \rightarrow \infty$ for a nonconstant polynomial $p$ with $p(A)=0$.

Proof. Suppose $T$ is compact and $p(T)$ is hyponormal for some nonconstant polynomial $p$. Use the Putnam inequality to see that $p(T)$ is a compact normal operator. Thus we can write $p(T)=0 \oplus S$ with respect to the decomposition $N(p(T)) \oplus \overline{R(p(T))}$, where $S$ is an injective compact normal operator. Write $T=\left(\begin{array}{cc}A & C \\ D & B\end{array}\right)$ with respect to the decomposition $N(p(T)) \oplus \overline{R(p(T))}$. Since $T$ commutes with $p(T)$, a straightforward calculation shows that $C S=S D=0$. But since $S$ is injective and has dense range we should have that $C=D=0$. Therefore $T=A \oplus B$ with $B S=S B$. Remember that $S$ is diagonalizable, i.e., $S=\sum_{n=1}^{\infty} \lambda_{n} P_{n}$, where $\left\{\lambda_{n}\right\}$ are the distinct nonzero eigenvalues of $p(T)$ and $P_{n}$ is the orthogonal projection of $\mathcal{H}$ onto $N\left(p(T)-\lambda_{n} I\right)$. Note that $P_{n}$ is of finite rank and $\lambda_{n} \rightarrow 0$. Therefore $T$ admits a direct sum $T=A \oplus B$, where $p(A)=0$ and $B=\bigoplus_{n=1}^{\infty} F_{n}$ with $F_{n}$ acting on $N\left(p(T)-\lambda_{n} I\right)$ for every $n=1,2 \cdots$. In this case $p\left(F_{n}\right)=\lambda_{n}$.

\section{REFERENCES}

1. S.K. Berberian, An extension of Weyl's theorem to a class of not necessarily normal operators, Michigan Math. J. 16 (1969), 273-279. MR 40:3335

2. S.K. Berberian, The Weyl spectrum of an operator, Indiana Univ. Math. J. 20 (1970), 529544. MR 43:5344

3. J.B. Conway and B.B. Morrel, Roots and Logarithms of bounded operators on Hilbert space, J. Funct. Anal. 70 (1987), 171-193. MR 87m:47044

4. L.A. Coburn, Weyl's theorem for nonnormal operators, Michigan Math. J. 13 (1966), 285288. MR 34:1846]

5. P.R. Halmos, A Hilbert Space Problem Book, Springer, New York, 1982. MR 84e:47001]

6. R.E. Harte, Fredholm, Weyl and Browder theory, Proc. Royal Irish Acad. 85A (2) (1985), 151-176. MR 87h:47029 
7. R.E. Harte, Invertibility and Singularity for Bounded Linear Operators, Dekker, New York, 1988. MR 89d:47001

8. R.E. Harte and W.Y. Lee, Another note on Weyl's theorem, Trans. Amer. Math. Soc. 349 (1997), 2115-2124. MR 98j:47024

9. W.Y. Lee and S.H. Lee, A spectral mapping theorem for the Weyl spectrum, Glasgow Math. J. 38(1) (1996), 61-64. MR 97c:47023

10. K.K. Oberai, On the Weyl spectrum (II), Illinois J. Math. 21 (1977), 84-90. MR 55:1102

11. C. Schmoeger, Ascent, descent and the Atkinson region in Banach algebras II, Ricerche di Matematica vol. XLII, fasc. ${ }^{\circ}$ (1993), 249-264. MR 95g:46093

12. H. Weyl, Über beschränkte quadratische Formen, deren Differenz vollsteig ist, Rend. Circ. Mat. Palermo 27 (1909), 373-392.

Department of Mathematics, Sungkyunkwan University, Suwon 440-746, Korea

Department of Mathematics, Sungkyunkwan University, Suwon 440-746, Korea

E-mail address: wylee@yurim.skku.ac.kr 